\title{
Notch1 signaling contributes to stemness in head and neck squamous cell carcinoma
}

\author{
Sang H Lee', Sung I Do², Hyun J Lee², Hyun J Kang ${ }^{3}$, Bon S Koo ${ }^{4}$ and Young C Lim³
}

Notch1 is associated with the initiation and progression of various solid tumors. However, the exact role of Notch1 expression in head and neck squamous cell carcinoma (HNSCC) remain unclear. We created cells ectopically expressing notch intracellular domain (NICD) from previously established HNSCC cells and examined self-renewal capacity and stem cell markers' expression compared with control cells. In addition, we knocked Notch1 down in primary spheres obtained from HNSCC tumor tissue and assessed the attenuation of stemness-associated traits in these cells in vitro and in vivo. Furthermore, we examined clinical relevance of Notch1 expression in HNSCC patients. Constitutive activation of NICD promoted the self-renewal capacity of HNSCC cells by activating sphere formation and increased the expression of stem cell markers such as Oct4, Sox2, and CD44. In contrast, Notch1 knockdown in primary HNSCC cancer stem cells (CSCs) attenuated CSC traits and augmented the chemosensitizing effects of cisplatin along with the decreased expression of almost all of $A B C$ transporter genes. In addition, Notch1 knockdown in HNSCC CSCs inhibited tumor formation and increased survival of mice in a xenograft model. Also, Notch1 acted upstream of canonical Wnt signaling in HNSCC cells. Finally, elevated Notch1 expression is associated with poor prognosis in patients with HNSCC. In conclusion, Notch1 may be a critical regulator of stemness in HNSCC cells, and inactivation of this pathway could be a potential targeted approach for the treatment of HNSCC.

Laboratory Investigation (2016) 96, 508-516; doi:10.1038/labinvest.2015.163; published online 29 February 2016

Head and neck squamous cell carcinoma (HNSCC) arises from the mucosal linings of the upper respiratory and digestive tracts and is the sixth leading cancer by incidence worldwide, with 600000 new cases being reported every year. ${ }^{1}$ Despite recent advances in the understanding of HNSCC progression and the development of novel therapeutic targets, the 5 -year survival rate for HNSCC patients has remained in the range of $50-60 \%$ for the past three decades. ${ }^{2}$ Therefore, development of novel strategies for HNSCC treatment is warranted to improve the survival outcome of this deadly disease.

Most solid tumors, including HNSCC, contain a small population of pluripotent cancer stem cells (CSCs). ${ }^{3}$ The CSC hypothesis states that CSCs possess unique features of self-renewal, unlimited proliferation, asymmetric cell division, and resistance to chemotherapy. Notably, CSCs are thought to have a major role in tumor relapse and metastatic spread, which are the common causes of high morbidity and, ultimately, death of the majority of patients with HNSCC. ${ }^{4}$ Therefore, a deeper understanding of the biological characteristics of HNSCC CSCs is an important goal, and the targeted elimination of these CSCs is part of a new conceptual framework for HNSCC treatment.

Notch signaling is attractive among several molecular targets because it is involved in a variety of cellular processes, such as cell proliferation, differentiation, and survival. ${ }^{5}$ Notch proteins are a family of heterodimeric transmembrane receptors composed of an extracellular domain responsible for ligand recognition, a transmembrane domain, and an intracellular domain involved in signal transduction. To date, four Notch receptors (Notch 1-4) and five ligands (Jagged-1, Jagged-2, Delta-1, Delta-3, and Delta-4) have been described in mammals. When a Notch receptor is triggered by a ligand, Delta-Serrate-Lag2 family members are expressed on neighboring cells, followed by successive proteolytic cleavage events in the transmembrane domain of the receptor, leading

\footnotetext{
'Department of Otorhinolaryngology - Head and Neck Surgery, Medical Research Institute, Kangbuk Samsung Hospital, Sungkyunkwan University School of Medicine, Seoul, Korea; ' 2 Department of Pathology, Kangbuk Samsung Hospital, Sungkyunkwan University School of Medicine, Seoul, Korea; ${ }^{3}$ Department of OtorhinolaryngologyHead and Neck Surgery, Research Institute of Medical Science, Konkuk University School of Medicine, Seoul, Korea and ${ }^{4}$ Department of Otolaryngology-Head and Neck Surgery, Cancer Research Institute, Research Institute for Medical Science, Chungnam National University College of Medicine, Daejeon, Korea

Correspondence: Professor YC Lim, MD, PhD, Department of Otorhinolaryngology-Head and Neck Surgery, Konkuk University School of Medicine, Hwayang-dong, Gwangin-gu, Seoul 143-752, Korea.
}

E-mail: yclim69@hanmail.net

Received 9 March 2015; revised 8 September 2015; accepted 16 September 2015 
a

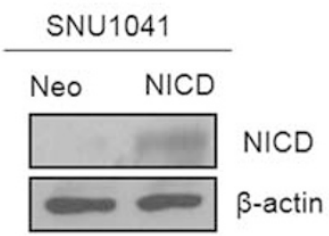

C

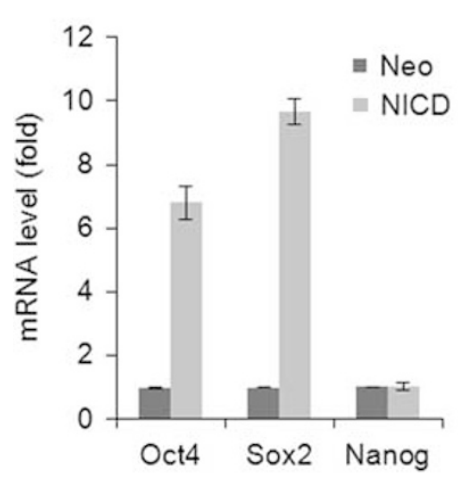

b

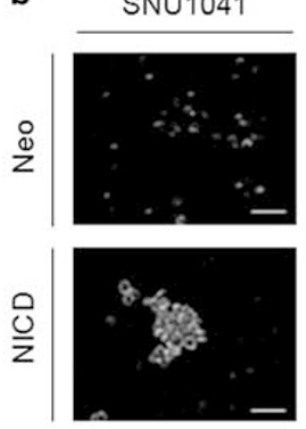

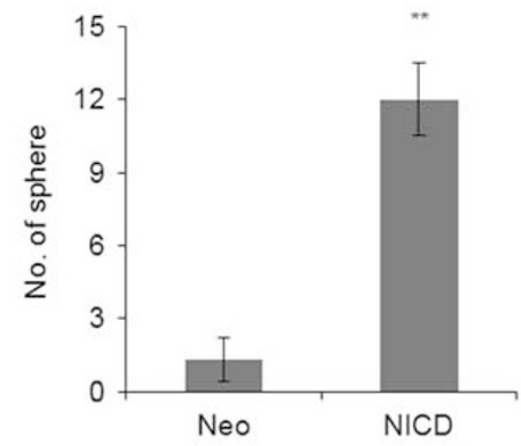

SNU1041

d
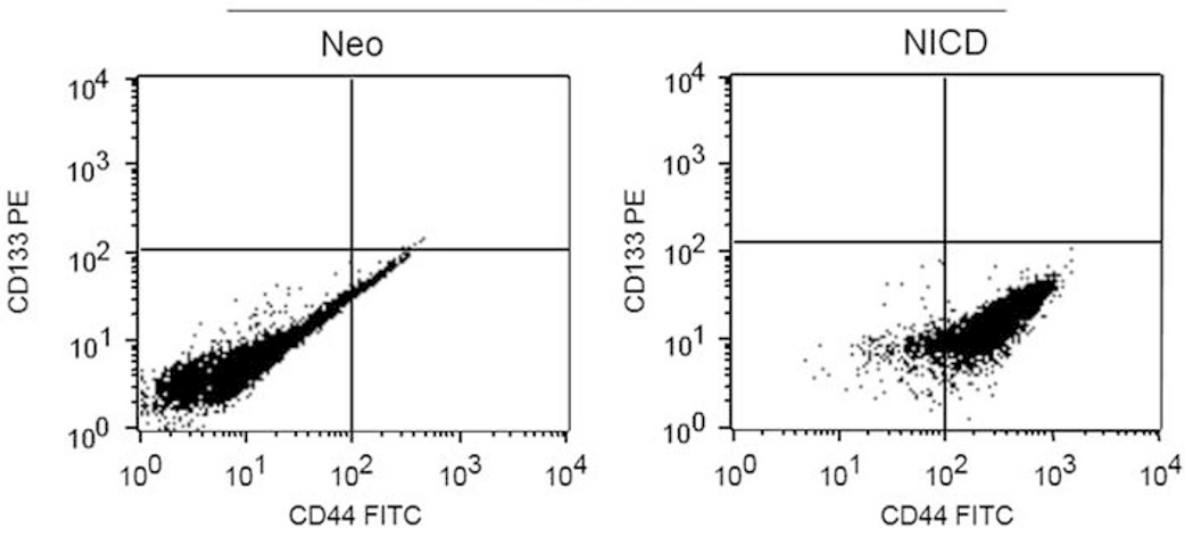

Figure 1 Differentiated HNSCC cells could acquire CSC phenotype through NICD activation. (a) NICD translational levels in the control vector-transduced SNU1041 cells and NICD-transduced SNU1041 cells by western blotting. (b) Sphere-forming capacity in the control vector-transduced SNU1041 cells and NICD-transduced SNU1041 cells. Bar, $10 \mu \mathrm{m}$. Data are expressed as mean \pm s.d. $(n=6)$. ${ }^{* * P}<0.01$. (c) mRNA levels of Oct4, Sox2, and Nanog, stemnessassociated genes, in the control vector-transduced SNU1041 cells and NICD-transduced SNU1041 cells. Data are expressed as mean \pm s.d. $(n=3)$.

(d) CD44 expression levels in the control vector-transduced SNU1041 cells and NICD-transduced SNU1041 cells by FACS analysis.

to the release of the Notch intracellular domain (NICD). ${ }^{6,7}$ NICD is released from the plasma membrane and translocates into the nucleus, where it forms a complex with the members of the CSL transcription factor family. This complex mediates the transcription of target genes such as Hes-1 and Hey-1, among others. ${ }^{6}$

Notch signaling has been shown to have an important role in cell fate determination, cell survival, and proliferation. ${ }^{8}$ Abnormal expression of Notch receptors has been observed in different types of epithelial malignant lesions. Moreover, the critical role of Notch signaling in angiogenesis and cisplatin resistance has been demonstrated in HNSCC. ${ }^{9,10}$ In addition, high expression of both Jagged 1 and Notch1 is associated with the worst prognosis in patients with HNSCC. ${ }^{11}$ Emerging evidence has also shown that Notch signaling is involved in the control of tumorigenesis and self-renewal of stem cells. ${ }^{12}$ However, two recent next-generation sequencing studies showed that Notch1 gene mutations, previously not known, have important roles in the carcinogenesis of HNSCC, suggesting the role of Notch1 as a tumor-suppressor gene. ${ }^{13,14}$ Thus the possible role and the consequence of the regulation of Notch1 signaling in HNSCC remain controversial. Nevertheless, systematic functional studies that elucidate the exact role of the Notch1 in HNSCC are hard to find, especially in HNSCC CSCs. In the present work, we examined whether Notch1 signaling affects the stem cell traits of HNSCC CSCs and the prognosis of patients with HNSCC. Our findings provide evidence that Notch1 has a critical role in regulating the characteristics of HNSCC CSCs.

\section{MATERIALS AND METHODS \\ Cell Lines and Reagent}

Human HNSCC cell line SNU 1041 was purchased from Korean Cell Line Bank and grown in DMEM in a humidified atmosphere with $5 \% \mathrm{CO}_{2}$ at $37^{\circ} \mathrm{C}$. HNSCC CSCs (K3, K4, and $\mathrm{K} 5$ ) was isolated and characterized from the primary surgical specimen of a HNSCC patient as previously described. $^{3}$ These cells were maintained in DMEM containing B27 (Invitrogen), N2 supplement (Invitrogen), basic fibroblast growth factor $(20 \mathrm{ng} / \mathrm{ml}$, R\&D systems, Minneapolis, MN, USA), and epidermal growth factor ( $20 \mathrm{ng} / \mathrm{ml}$, R\&D systems). For chemoresistance assay, cisplatin was purchased from Sigma (Sigma-Aldrich, St Louis, MO, USA). Primary antibodies were purchased as follows: Notch1, CD44, Oct4, Sox2, Ki-67, and $\beta$-catenin from Santa Cruz Biotechnology (Santa Cruz, CA, USA), and 

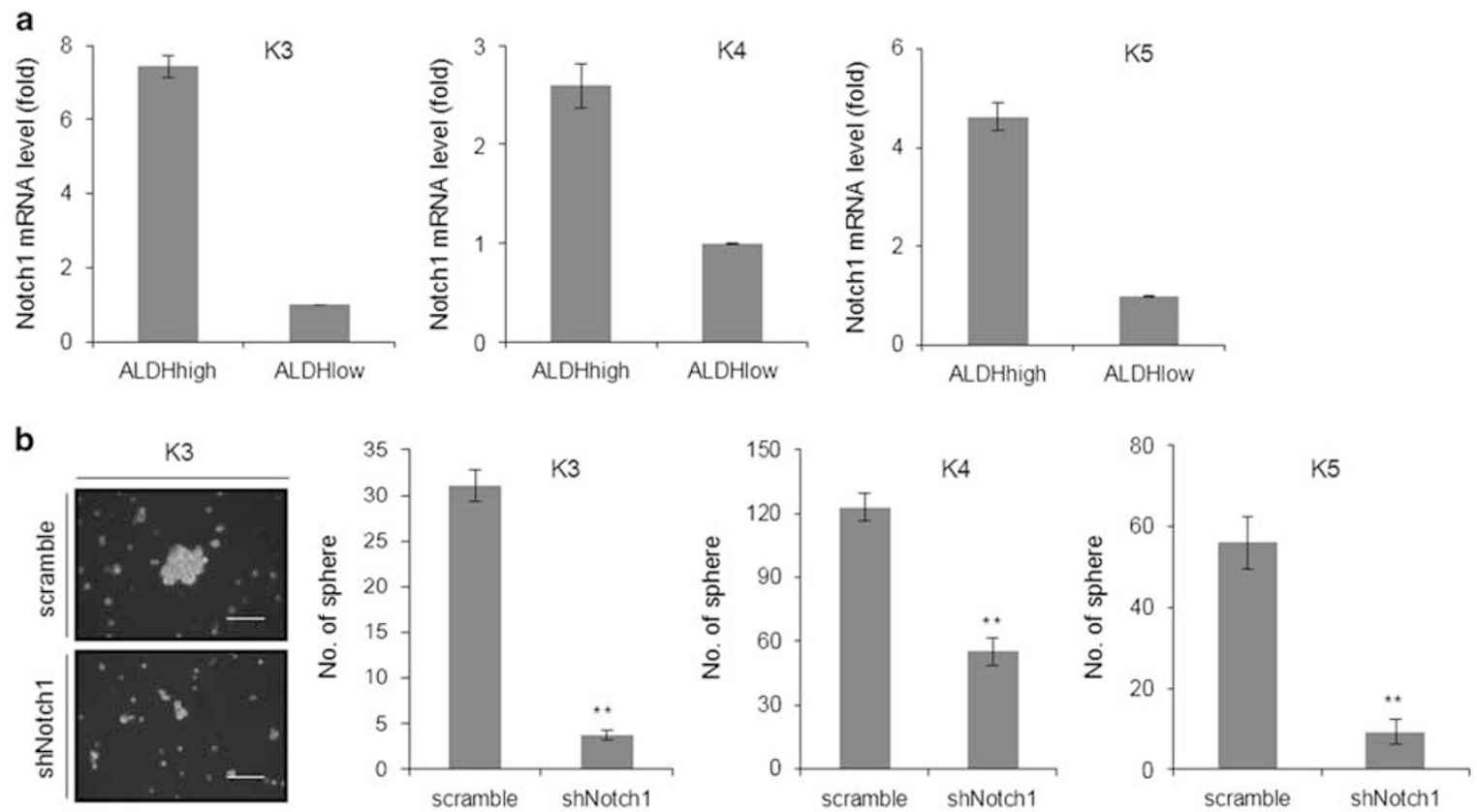

C

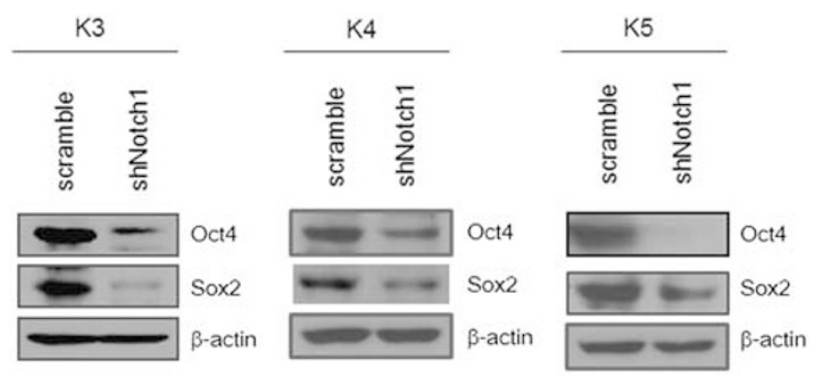

d
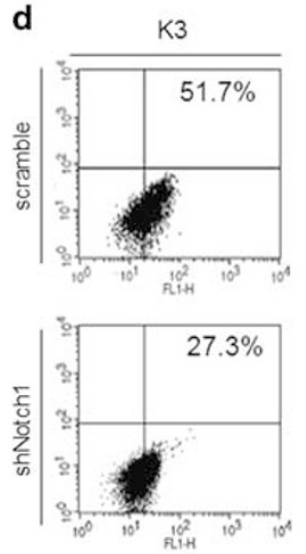
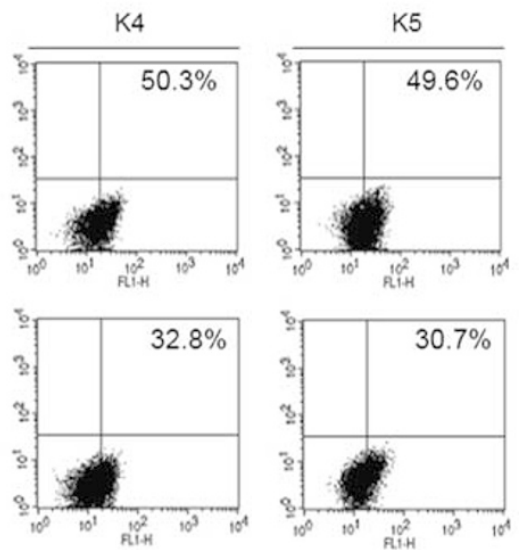

Figure 2 Knockdown of Notch1 suppresses cancer stem cell traits of HNSCC CSC. (a) The mRNA levels of Notch 1 in ALDH ${ }^{\text {high }}$ - and ALDH ${ }^{\text {low }}-$ HNSCC CSCs. Data are expressed as mean \pm s.d. $(N=3)$. ALDH, aldehyde dehydrogenase. (b) Sphere-forming ability of HNSCC CSC-shScramble and HNSCC CSC-shNotch1 cells in serum-free conditions. Bar, $10 \mu \mathrm{m}$, Data are expressed as mean \pm s.d. $(N=3)$. ${ }^{* *} P<0.01$. (c) The protein levels of stemness-associated genes (Oct4 and Sox2) in HNSCC CSC-shScramble and HNSCC CSC-shNotch1 cells determined by western blotting. (d) CD44 expression in HNSCC CSC-shScramble and HNSCC CSC-shNotch1 cells were determined by FACS analysis.

NICD from Cell signaling (Denver, MA, USA). Secondary antibodies, anti-rabbit IgG or anti-mouse IgG, were purchased from Jackson ImmunoResearch Laboratories (West Grove, PA, USA).

Full materials and methods are available in the Supplementary Methods.

\section{RESULTS}

\section{Constitutive Activation of NICD Enhances HNSCC Cell Stemness}

We initiated an in vitro study to investigate whether constitutive activation of Notch1 signaling could promote HNSCC CSC traits. For this, we generated NICD-overexpressing SNU1041 (SNU1041-NICD) and control vector-transducing
SNU1041 cells by retroviral gene transfer, which were confirmed by western blotting analysis (Figure 1a). Additionally, we confirmed that the expression levels of Hes1 and Heyl, downstream molecules of NICD, were significantly upregulated in SNU1041-NICD cells (Supplementary Figure S1). Next, we performed an in vitro sphere-forming assay to compare the $\mathrm{s}$ elf-renewal ability of SNU1041-NICD cells with that of SNU1041-control cells. We found that the sphere-forming ability of SNU1041-NICD cells was significantly higher than that of SNU1041-control cells in serum-free condition (Figure 1b). Several markers, including Oct4, Sox2, Nanog, and CD44, are associated with CSC properties. Therefore, we subsequently analyzed the expression of these stem cell markers in SNU1041-NICD cells compared with that in 

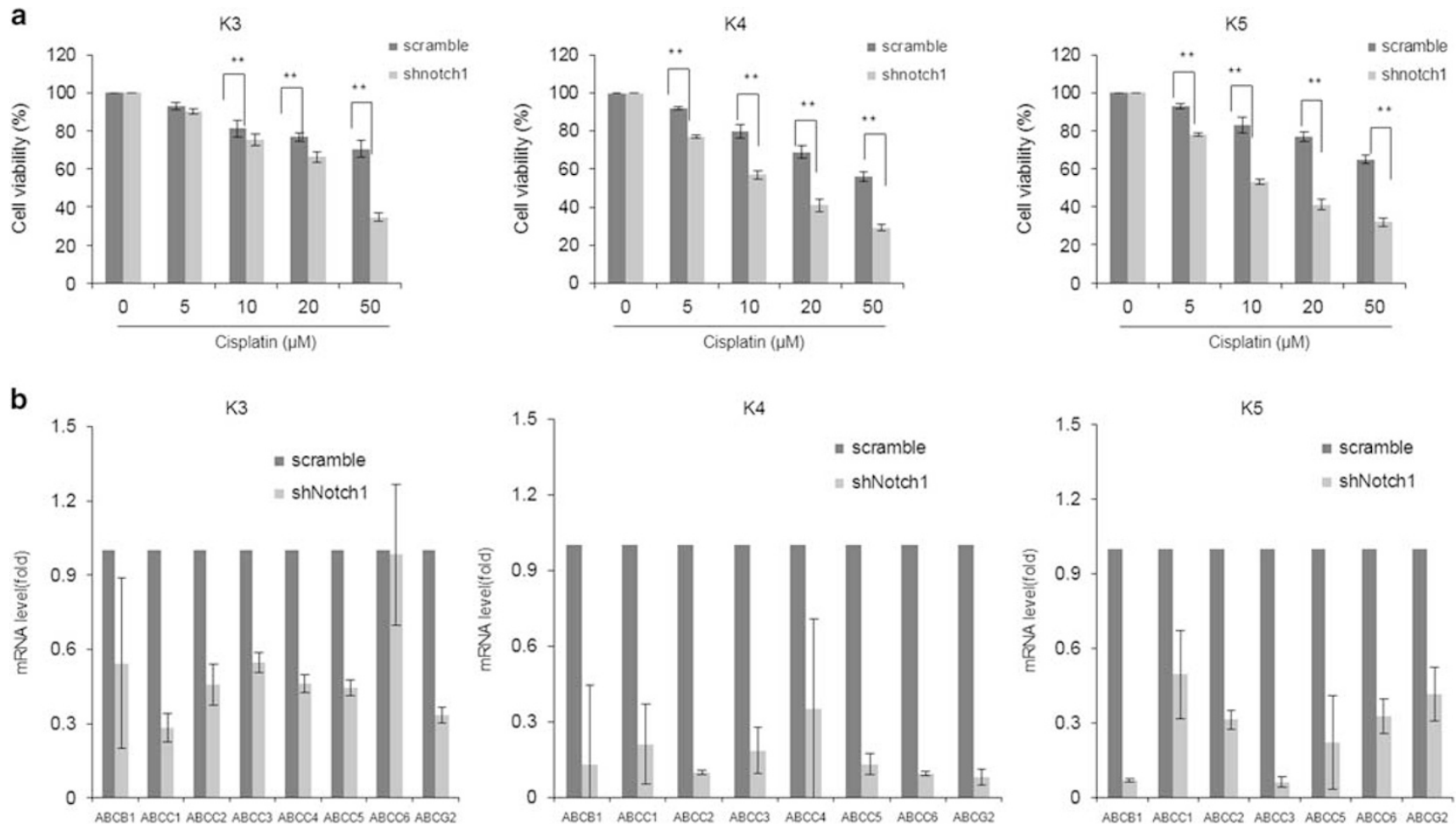

Figure 3 Knockdown of Notch1 enhances the sensitivity of HNSCC CSCs to cisplatin. (a) MTT assay after cisplatin administration with various concentrations in HNSCC CSC-shScramble and HNSCC CSC-shNotch1 cells. Data are expressed as mean \pm s.d. $(N=3)$. ${ }^{*} P<0.01$. (b) mRNA levels of different ABC transporters in HNSCC CSC-shScramble and HNSCC CSC-shNotch1 cells. Data are expressed as mean \pm s.d. $(N=3)$.

SNU1041-control cells. Transcriptional levels of Oct4 and Sox2 were considerably increased in SNU1041-NICD cells (Figure 1c). In addition, flow cytometry analysis to quantify CD44 expression, a marker of HNSCC CSCs, showed that CD44+ cells were considerably increased from 2.54 to $14.47 \%$ in SNU1041-NICD cells (Figure 1d). Collectively, activation of Notch1 signaling appears to enhance the stemness phenotype of HNSCC cells.

\section{Knockdown of Notch1 Attenuates HNSCC CSC Traits In Vitro}

CSCs have been identified in human HNSCC using aldehyde dehydrogenase (ALDH) activity. We therefore compared the Notch1 mRNA levels of ALDH ${ }^{\text {high }}$ and ALDH ${ }^{\text {low }}$ cells in HNSCC CSCs and observed increased Notch1 mRNA levels in $\mathrm{ALDH}^{\text {high }}$ cells compared with ALDH ${ }^{\text {low }}$ cells (Figure 2a). To further determine the regulatory role of wild-type Notch1 in HNSCC CSCs, we assessed the effect of Notch1 downregulation on cultured HNSCC CSCs. For this study, HNSCC CSCs were transduced with control-shRNA or Notch1-specific shRNA by lentiviral vectors. Western blotting analysis confirmed the efficient knockdown of Notch1 expression (Supplementary Figure S2A). We also confirmed the downregulated transcription of the Notch1 target genes Hes1 and Heyl in HNSCC CSC-shNotch1 cells
(Supplementary Figure S2B). Contrary to Notch1 activation, Notch1 knockdown decreased the sphere-forming ability of HNSCC CSCs (Figure 2b). Western blotting analysis showed that Notch1 knockdown led to decreased protein levels of CSC markers such as Oct and Sox2 (Figure 2c). FACS analysis showed that CD44 expression was also decreased in HNSCC CSC-shNotch1 cells (Figure 2d).

\section{Knockdown of Notch 1 Enhances the Sensitivity of HNSCC CSCs to Cisplatin by Suppressing ABCC2 and ABCG2 Transporter Gene Expression}

Next we conducted a methyl thiazolyl tetrazolium (MTT) assay to evaluate the effect of Notch1 knockdown on the chemosensitivity of HNSCC CSCs. The MTT assay revealed that Notch1 knockdown decreased the number of viable HNSCC CSCs after cisplatin treatment, compared with that of control cells (Figure 3a). The chemoresistance of CSCs could be attributed to their increased ability to exclude exogenous and endogenous toxic materials via ATP-binding cassette $(\mathrm{ABC})$ transporters. Therefore, we investigated the changes in ABC transporter levels in HNSCC CSCs after Notch1 knockdown and observed that Notch1 knockdown decreased the mRNA expression levels of almost all $\mathrm{ABC}$ transporter genes in HNSCC CSCs (Figure 3b). 
a

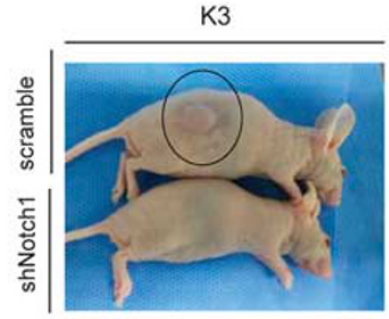

\begin{tabular}{|c|r|}
\hline Injected cells & Tumor formation \\
\hline scramble \\
\hline $10^{3}$ & $3 / 5$ \\
\hline $10^{4}$ & $5 / 5$ \\
\hline $10^{5}$ & $5 / 5$ \\
\hline shNotch1 & \\
\hline $10^{3}$ & $0 / 5$ \\
\hline $10^{4}$ & $0 / 5$ \\
\hline $10^{5}$ & $2 / 5$ \\
\hline
\end{tabular}

d

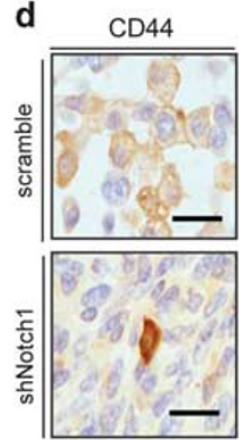

b

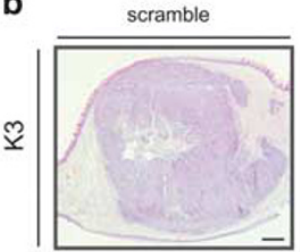

shNotch1

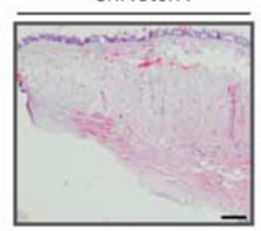

c

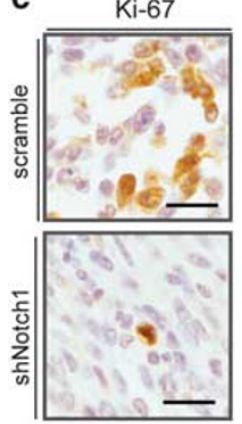

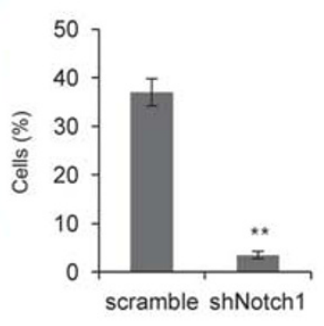

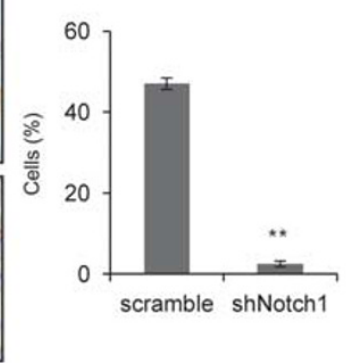

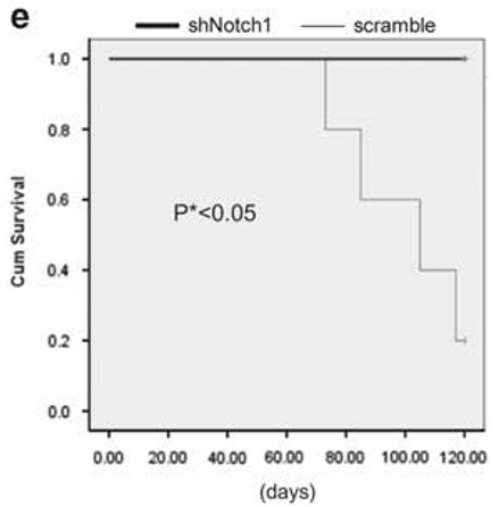

Figure 4 Targeting Notch1 suppresses HNSCC CSC tumor growth and increases survival of mice bearing HNSCC CSC xenografts. (a) In vivo propagation of human head and neck cancer in nude mice by either HNSCC CSC-shScramble or HNSCC CSC-shNotch1cells (right panel). Representative photos showing tumor in a mouse at the cell injection site (left panel). (b) Hematoxylin and eosin slides of tongue tumor tissue generated by injection of HNSCC CSC-shScramble and HNSCC CSC-shNotch1. Bar, $500 \mu \mathrm{m}$, Data are expressed as mean \pm s.d. ( $N=5$ ). (c) Percentage of positive cells for Ki-67, a proliferation marker, in xenograft tumor tissue generated by injection of HNSCC CSC-shScramble and HNSCC CSC-shNotch1. Bar, $20 \mu$ m, Data are expressed as mean \pm s.d. $(N=3),{ }^{* *} P<0.05$. (d) Percentage of positive cells for CD44, a putative HNSCC CSC marker, in xenograft tumor tissue generated by injection of HNSCC CSC-shScramble and HNSCC CSC-shNotch1. Bar, $20 \mu \mathrm{m}$, Data are expressed as mean $\pm \mathrm{s} . \mathrm{d}$. $(N=3)$, ${ }^{* *} P<0.05$. (e) Kaplan-Meier survival curves of mice orthotopically implanted with HNSCC CSC-shScramble and HNSCC CSC-shNotch1 cells $(n=5)$. ${ }^{*} P<0.05$.

\section{Knockdown of Notch1 Induces Antitumor Effects in a Xenograft Model of HNSCC CSCs}

To validate the in vitro finding that suppression of Notch1 decreased the stem cell traits of HNSCC CSCs in vivo, we investigated the inhibitory effects of Notch1 knockdown on HNSCC CSC-mediated tumor initiation in a mouse xenograft model. We subcutaneously injected varying numbers of HNSCC CSC-shNotch1 cells $\left(10^{3}, 10^{4}\right.$, or $\left.10^{5}\right)$ and HNSCC CSC-scramble shRNA cells $\left(10^{3}, 10^{4}\right.$, or $\left.10^{5}\right)$ in $\mathrm{BALB} / \mathrm{c}$ mice. As shown in Figure $4 \mathrm{a}$, even $10^{3}$ HNSCC CSC-scramble shRNA cells were sufficient to initiate tumor formation 6 weeks after injection; however, only 40\% (2 out of 5) of mice injected with $10^{5}$ HNSCC CSC-shNotch1 cells exhibited visible tumor formation. In an orthotopic model, the injection of HNSCC CSC-scramble shRNA cells produced tongue tumors in all of the injected mice $(n=5)$, but HNSCC CSC-shNotch1 cells did not (Figure 4b). Next, we examined whether Notch1 knockdown affected the cell proliferation rate using Ki-67 staining in sections from the xenografted tumors. We observed that Notch1 knockdown significantly decreased the expression of Ki-67-positive cells (Figure 4c) and CD44-positive cells, putative markers of HNSCC CSCs (Figure 4d). Finally, we compared the survival rates of mice injected with HNSCC CSC-shNotch1 cells or HNSCC CSC-scramble shRNA and observed a significant increase in cumulative survival in mice bearing tumors formed from HNSCC CSC-shNotch1 cells compared with those bearing tumors formed from HNSCC CSC-scramble shRNA cells (Figure 4e). Taken together, our findings suggested that Notch1 has a critical role in tumor propagation of HNSCC CSCs in a xenograft model.

\section{Notch1 Signaling Acts Upstream of Canonical Wnt Signaling in HNSCC Cells}

We previously observed that canonical Wnt signaling maintains the stem cell characteristics of HNSCC cells. ${ }^{15}$ The stem-like phenotype of cells with Notch1 activation led us to examine the relationship between the Notch1 and Wnt signaling pathways. Nuclear $\beta$-catenin levels, an indicator of canonical Wnt signaling activation, increased or decreased with Notch1 overexpression and suppression, respectively (Figure 5a and Supplementary Figure S3). Relative to 
a
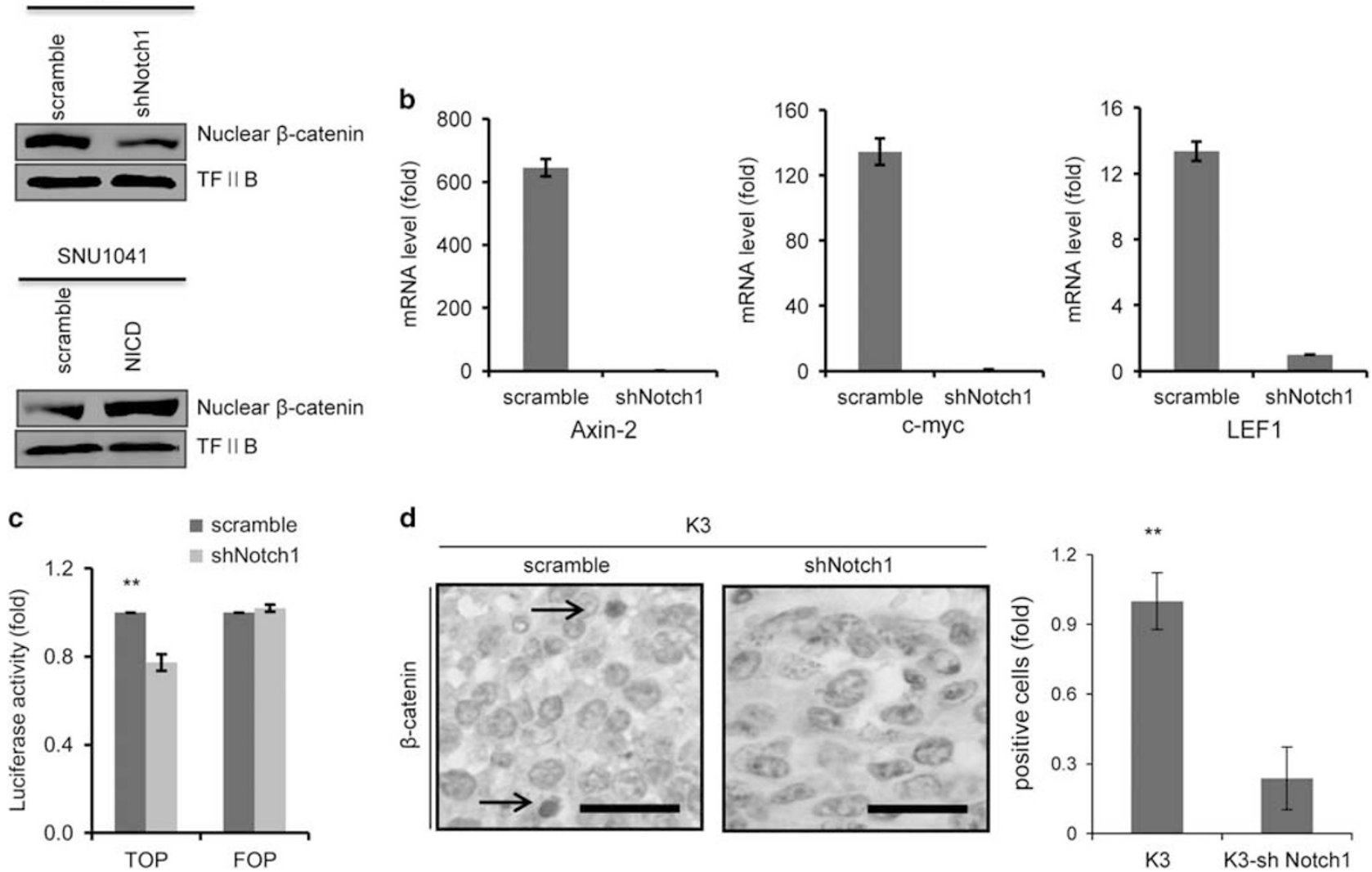

Figure 5 Notch1 signaling acts upstream of canonical Wnt signaling in HNSCC cells. (a) The protein levels of nuclear $\beta$-catenin in HNSCC CSCshScramble and HNSCC CSC-shNotch1 cells (upper) and SNU1041-NICD and SNU1041-control cells (lower). (b) mRNA levels of different target genes of nuclear $\beta$-catenin (Axin-2, c-myc, and LEF1) in HNSCC CSC-shScramble and HNSCC CSC-shNotch1 cells. Data are expressed as mean \pm s.d. ( $N=3$ ). (c) Reporter gene assay of TOP/FOPlash of HNSCC CSC-shScramble and HNSCC CSC-shNotch1 cells. Data are expressed as mean $\pm s . d$. ( $N=3$ ). **P $<0.05$. (d) Percentage of positive cells for nuclear $\beta$-catenin in xenograft tumor tissue generated by injection of HNSCC CSC-shScramble and HNSCC CSC-shNotch1. Arrow indicates nuclear $\beta$-catenin-positive cells. Data are expressed as mean \pm s.d. $(N=3),{ }^{* *} P<0.05$.

vector-transduced controls, the expression levels of Axin-2, c-Myc, and LEF-1, various target genes of $\beta$-catenin, as well as TOP luciferase reporter activity were significantly decreased in Notch1 knocked down HNSCC CSCs (Figures 5b and c). In addition, immunohistochemical examination of nuclear $\beta$-catenin in tumors derived from the in vivo mouse model revealed significantly decreased nuclear $\beta$-catenin expression in tumor tissues derived from shNotch1-HNSCC CSCs injection compared with those derived from control-HNSCC CSCs (Figure 5d). To confirm Wnt signaling activation as the mechanism mediating Notch1 effects in HNSCC CSCs, we studied whether ectopic expression of $\beta$-catenin rescues the decreased CSC phenotypes caused by Notch1 downregulation in HNSCC stem-like cells. First, we transfected $\beta$-catenin-overexpressing vector into HNSCC stem-like cells with Notch1- shRNA (Supplementary Figure S4A). Forced expression of $\beta$-catenin in shNotch1 HNSCC stem-like cells not only restored tumor sphere formation and but also rescued chemoresistance (Supplementary Figure S4B and $\mathrm{S} 4 \mathrm{C}$ ). In addition, ectopic expression of $\beta$-catenin increased cell proliferation capacity (Supplementary
Figure S4D). Taken together, these results suggest that the Notch1 pathway maintains partly the stem cell traits of HNSCC CSCs through Wnt signaling.

\section{Notch1 Expression can Predict the Prognosis of Patients with HNSCC}

To explore the predictive value of Notch 1 expression levels on the prognosis of HNSCC patients, we analyzed the survival data from 67 patients with HNSCC based on Notch1 expression. The clinicopathological features of these patients are summarized in Table 1. Notch1 was poorly expressed in 26 samples and highly expressed in 41 samples (Supplementary Figure S5). Notch1 expression was significantly correlated with lymph node metastasis and tumor recurrence $(P<0.05$; Table 1$)$. Notch1 expression and Kaplan-Meier survival estimates for patients with HNSCC are shown in Figure 6, suggesting that patients with increased Notch1 expression had a significantly worse prognosis than those with decreased expression $(P<0.05)$. Altogether, these results suggest that Notch1 expression significantly affects the prognosis of patients with HNSCC. 
Table 1 Relationship between Notch1 expression and clinicopathological parameters in patients with HNSCC $(n=67)$

\begin{tabular}{lll}
\hline Variables & No. of cases & $\begin{array}{c}\text { Notch1 } \\
\text { expression }\end{array}$ \\
& Low High & \\
\hline
\end{tabular}

(a) Univariate analysis

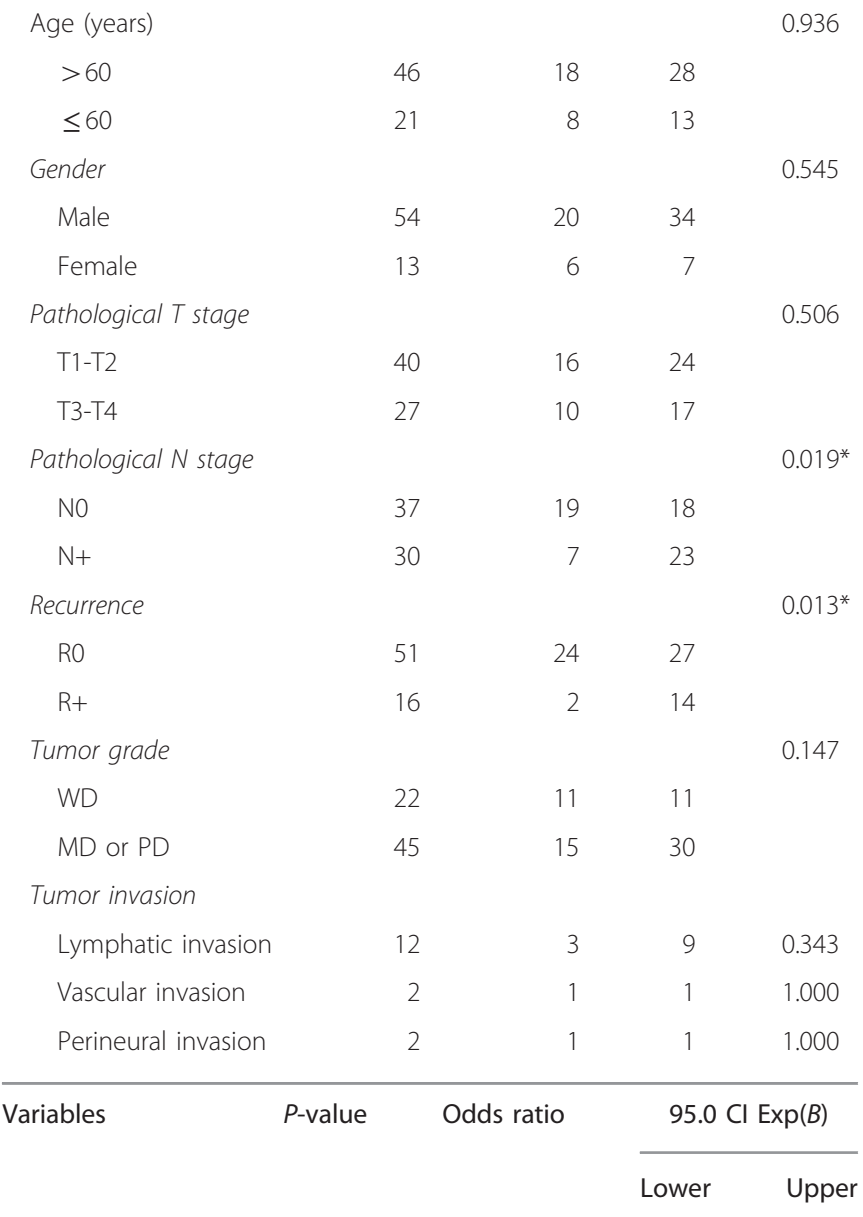

(b) Multivariate analysis

\begin{tabular}{lllll} 
Pathological N+ & $0.030^{*}$ & 4.135 & 1.151 & 14.857 \\
Recurrence & $0.018^{*}$ & 7.726 & 1.426 & 41.863 \\
\hline
\end{tabular}

${ }^{*} P<0.05$.

\section{DISCUSSION}

Tumors are composed of a heterogeneous group of cells. At least two different models, the stochastic model and the hierarchy model, have been proposed to explain the heterogeneity of tumor cells. ${ }^{16,17}$ The hierarchy (or CSC) model suggests that tumors are composed of a heterogeneous group of cells that have arisen from stem-like precursors. CSCs are characterized by their tumorigenic properties and their ability to self-renew, form differentiated progeny, and develop resistance to therapy. Recently, we demonstrated a number of primary HNSCC-driven squamospheres that possess the general properties of CSCs, including self-renewal, stem cell marker expression, aberrant differentiation, tumor-initiating potential, and chemoresistance with an increased side population. ${ }^{3}$ The origin of CSCs is not fully understood; however, data suggest that they originate from normal stem or progenitor cells or, possibly, from other cancer cells. ${ }^{18}$ Tumors composed of small populations of CSCs and large numbers of differentiated tumor cells may be particularly susceptible to combination drug regimens that target each cell population. Therapeutic targeting of both CSCs and bulk tumor populations may provide a strategy to suppress tumor regrowth. ${ }^{18}$

Tumor relapse and metastasis remain major obstacles to improving overall cancer survival, and CSCs are considered a major cause of tumor relapse after conventional therapy because of their resistance to chemotherapy and radiotherapy modalities. ${ }^{19,20}$ The slow growth rate and chemoresistant characteristics suggest that CSCs may survive routine chemotherapy, only to reinitiate tumor growth at a later point in time. ${ }^{21}$ Several mechanisms are involved in CSC chemoresistance, including the expression of the ATP-binding cassette superfamily of active drug transporters. ${ }^{22}$ Members of this transmembrane superfamily act as unidirectional cellular pumps and have been linked with multidrug resistance. Resistance to anticancer drugs could be attributed to the reduced levels of drug accumulation within CSCs.

Notch signaling has a critical role in cellular developmental pathways, including proliferation, differentiation, and apoptosis. ${ }^{8}$ It also contributes to cancer progression by activating transcription factors that promote cell survival, motility, and tumor angiogenesis. ${ }^{23}$ Emerging lines of evidence have suggested that cancers can grow from CSCs, and the Notch pathway is believed to be deregulated, leading to uncontrolled self-renewal of CSCs that generate tumors. Notch may drive tumor growth through the generation or expansion of CSCs. ${ }^{24,25}$ A growing body of literature suggests the biological importance of Notch signaling in cancer and CSCs, further suggesting that inactivation of Notch signaling by novel approaches could be useful for cancer therapy.

The Notch gene is abnormally activated in several human malignancies. Upregulated expression of Notch receptors and their ligands in cervical, lung, colon, renal, and pancreatic cancers have been reported. ${ }^{7,26}$ It has recently been demonstrated that Notch1 mutations are frequently found in HNSCC, suggesting a potential role for this pathway in the biology of CSCs and in the etiology of head and neck cancer. ${ }^{13}$ Zhang et al. ${ }^{27}$ reported high expression levels of Notch receptors and ligands in tongue cancer and suggested that Notch signaling may control the differentiation and proliferation of tongue carcinoma cells. Zeng et al. ${ }^{10}$ reported that HNSCC may utilize the Notch signaling pathway to promote tumor angiogenesis in vivo, and that the level of Jagged 1 expression is associated with the development of 
a

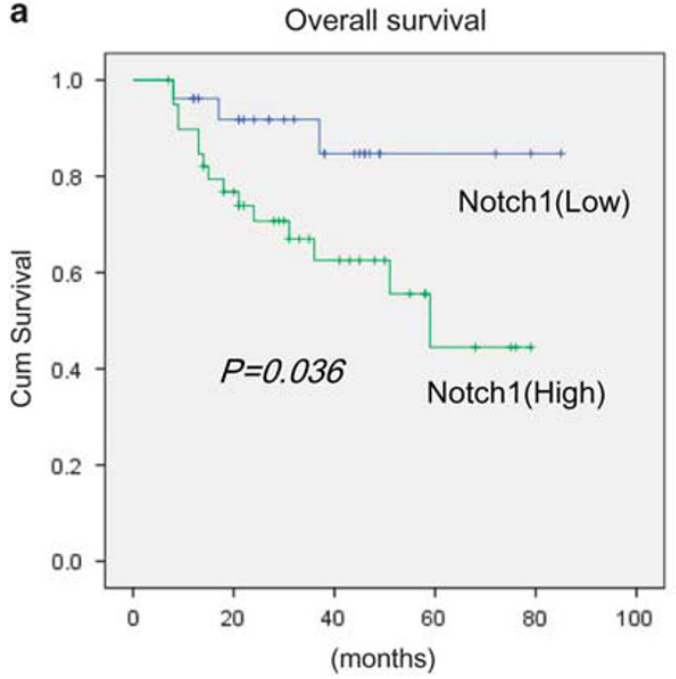

b

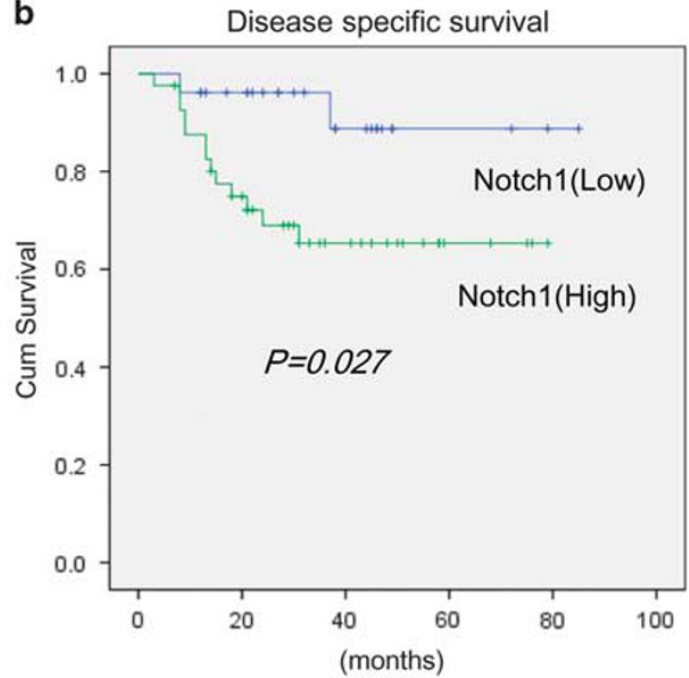

Figure 6 Notch1 expression level is correlated with survival of patients with HNSCC. (a) Overall survival using Kaplan-Meier analysis based on Notch1 expression in patients with HNSCC $(n=67)$. (b) Disease-specific survival using Kaplan-Meier analysis based on Notch1 expression in patients with HNSCC $(n=67)$.

HNSCC. Gu et al. ${ }^{9}$ reported that Notch1 expression is associated with cisplatin resistance in HNSCC. In addition, Lin et al. ${ }^{11}$ showed that high co-expression of Jagged-1 and Notch1 signaling is associated with the worst prognosis in patients with HNSCC. Thus Notch signaling has an important role in the carcinogenesis and prognosis of HNSCC. However, to the best of our knowledge, the possible role and the consequence of the regulation of Notch signaling in HNSCC CSCs has never been examined.

In this study, we investigated the role of Notch1 signaling in HNSCC and HNSCC CSCs. Activation of Notch1 signaling is correlated with cell proliferation of HNSCC through influence on cell cycle progression. Furthermore, constitutive activation of NICD promoted CSC traits in differentiated HNSCC cells. In contrast, downregulation of Notch1 signaling led to attenuation of CSC traits in HNSCC CSCs and enhanced the chemosensitivity of cisplatin to HNSCC CSCs by suppressing $\mathrm{ABCC} 2$ and $\mathrm{ABCG} 2$ transporter gene expression. Interestingly, enhanced stemness after Notch1 signaling activation in HNSCC CSCs is associated with crosstalk with the canonical Wnt signaling pathway. Finally, high Notch1 expression was associated with poor prognosis in patients with HNSCC. Collectively, our data suggest that Notch1 signaling, as a major target for HNSCC, has an important role in HNSCC and HNSCC CSCs.

Current chemotherapeutic agents may target and kill the overall population of differentiated tumor cells, which constitute the bulk of the tumor. CSCs, with slower and less abundant proliferation, express drug transporters and thus appear to be relatively drug resistant. Therefore, they are spared, ultimately inducing tumor relapse after completion of treatment. The development of new therapeutic approaches targeting CSCs has become a major issue in cancer biology. With these considerations, our results suggest that the Notch1 pathway may be an attractive target for the treatment of HNSCC.

Supplementary Information accompanies the paper on the Laboratory Investigation website (http://www.laboratoryinvestigation.org)

\section{ACKNOWLEDGMENTS}

This work was supported by the National Research Foundation of Korea (NRF) grant funded by the Korean government (MEST) (Grant numbers 2012R1A2A2A01046214 and 2015R1A2A2A01006688 to Lim YC) and the Samsung Biomedical Research Institute grant (SH Lee).

\section{DISCLOSURE/CONFLICT OF INTEREST}

The authors declare no conflict of interest.

1. Kamangar F, Dores GM, Anderson WF. Patterns of cancer incidence, mortality, and prevalence across five continents: defining priorities to reduce cancer disparities in different geographic regions of the world. J Clin Oncol 2006;24:2137-2150.

2. Carvalho $A L$, Nishimoto $I N$, Califano JA et al. Trends in incidence and prognosis for head and neck cancer in the United States: a site-specific analysis of the SEER database. Int J Cancer 2005;114:806-816.

3. Lim YC, Oh SY, Cha YY et al. Cancer stem cell traits in squamospheres derived from primary head and neck squamous cell carcinomas. Oral Oncol 2011;47:83-91.

4. Zhang Z, Filho MS, Nor JE. The biology of head and neck cancer stem cells. Oral Oncol 2012;48:1-9.

5. Wang Z, Li Y, Banerjee $S$ et al. Exploitation of the Notch signaling pathway as a novel target for cancer therapy. Anticancer Res 2008;28: 3621-3630.

6. Miele L. Notch signaling. Clin Cancer Res 2006;12:1074-1079.

7. Miele L, Miao H, Nickoloff BJ. NOTCH signaling as a novel cancer therapeutic target. Curr Cancer Drug Targets 2006;6:313-323.

8. Artavanis-Tsakonas S, Rand MD, Lake RJ. Notch signaling: cell fate control and signal integration in development. Science 1999;284: 770-776.

9. Gu F, Ma Y, Zhang Z et al. Expression of Stat3 and Notch1 is associated with cisplatin resistance in head and neck squamous cell carcinoma. Oncol Rep 2010;23:671-676.

10. Zeng Q, Li S, Chepeha DB et al. Crosstalk between tumor and endothelial cells promotes tumor angiogenesis by MAPK activation of Notch signaling. Cancer Cell 2005;8:13-23. 
11. Lin JT, Chen MK, Yeh KT et al. Association of high levels of Jagged-1 and Notch-1 expression with poor prognosis in head and neck cancer. Ann Surg Oncol 2010;17:2976-2983.

12. Dontu G, Jackson KW, McNicholas E et al. Role of Notch signaling in cell-fate determination of human mammary stem/progenitor cells. Breast Cancer Res 2004;6:R605-R615.

13. Agrawal N, Frederick MJ, Pickering CR et al. Exome sequencing of head and neck squamous cell carcinoma reveals inactivating mutations in NOTCH1. Science 2011;333:1154-1156.

14. Stransky N, Egloff AM, Tward AD et al. The mutational landscape of head and neck squamous cell carcinoma. Science 2011;333:1157-1160.

15. Lee $\mathrm{SH}, \mathrm{Koo} \mathrm{BS}, \mathrm{Kim} \mathrm{JM}$ et al. Wnt/ß-catenin signalling maintains selfrenewal and tumourigenicity of head and neck squamous cell carcinoma stem-like cells by activating Oct4. J Pathol 2014;234: 99-107.

16. Campbell LL, Polyak K. Breast tumor heterogeneity: cancer stem cells or clonal evolution? Cell Cycle 2007;6:2332-2338.

17. Reya T, Morrison SJ, Clarke MF et al. Stem cells, cancer, and cancer stem cells. Nature 2001;414:105-111.

18. Takebe N, Harris PJ, Warren RQ et al. Targeting cancer stem cells by inhibiting Wnt, Notch, and Hedgehog pathways. Nat Rev Clin Oncol 2011;8:97-106.
19. Dean $M$, Fojo $T$, Bates $S$. Tumour stem cells and drug resistance Nat Rev Cancer 2005;5:275-284.

20. Dick JE. Stem cell concepts renew cancer research. Blood 2008;112 4793-4807.

21. Frank NY, Schatton T, Frank MH. The therapeutic promise of the cance stem cell concept. J Clin Invest 2010;120:41-50.

22. Frank NY, Margaryan A, Huang $Y$ et al. ABCB5-mediated doxorubicin transport and chemoresistance in human malignant melanoma. Cancer Res 2005;65:4320-4333.

23. Wang Z, Li Y, Banerjee $\mathrm{S}$ et al. Emerging role of Notch in stem cells and cancer. Cancer Lett 2009;279:8-12.

24. Wilson A, Radtke F. Multiple functions of Notch signaling in self-renewing organs and cancer. FEBS Lett 2006;580:2860-2868.

25. Kakarala M, Wicha MS. Implications of the cancer stem-cell hypothesis for breast cancer prevention and therapy. J Clin Oncol 2008;26: 2813-2820.

26. Wang $Z$, Banerjee $S$, Li Y et al. Down-regulation of notch-1 inhibits invasion by inactivation of nuclear factor-kappaB, vascular endothelial growth factor, and matrix metalloproteinase-9 in pancreatic cancer cells. Cancer Res 2006;66:2778-2784.

27. Zhang TH, Liu HC, Zhu LJ et al. Activation of Notch signaling in human tongue carcinoma. J Oral Pathol Med 2011;40:37-45. 